\title{
LONGER CYCLES IN ESSENTIALLY 4-CONNECTED PLANAR GRAPHS
}

\author{
IGOR FABRICI $^{1,2, a}$, JOCHEN HARANT ${ }^{1, b}$ \\ SAmuel Mohr ${ }^{1,3, b}$ And Jens M. SChMidt ${ }^{1,3, b}$ \\ ${ }^{a}$ Pavol Jozef Šafárik University \\ Institute of Mathematics, Košice, Slovakia \\ ${ }^{b}$ Ilmenau University of Technology \\ Department of Mathematics, Ilmenau, Germany \\ e-mail: igor.fabrici@upjs.sk \\ jochen.harant@tu-ilmenau.de \\ samuel.mohr@tu-ilmenau.de \\ jens.schmidt@tu-ilmenau.de
}

\begin{abstract}
A planar 3-connected graph $G$ is called essentially 4-connected if, for every 3-separator $S$, at least one of the two components of $G-S$ is an isolated vertex. Jackson and Wormald proved that the length $\operatorname{circ}(G)$ of a longest cycle of any essentially 4-connected planar graph $G$ on $n$ vertices is at least $\frac{2 n+4}{5}$ and Fabrici, Harant and Jendrol improved this result to $\operatorname{circ}(G) \geq \frac{1}{2}(n+4)$. In the present paper, we prove that an essentially 4-connected planar graph on $n$ vertices contains a cycle of length at least $\frac{3}{5}(n+2)$ and that such a cycle can be found in time $O\left(n^{2}\right)$.
\end{abstract}

Keywords: essentially 4-connected planar graph, longest cycle, circumference, shortness coefficient.

2010 Mathematics Subject Classification: 05C38, 05C10.

\section{REFERENCES}

\footnotetext{
${ }^{1}$ Partially supported by DAAD, Germany (as part of BMBF) and by the Ministry of Education, Science, Research and Sport of the Slovak Republic within the project 57320575.

${ }^{2}$ Partially supported by Science and Technology Assistance Agency under the contract No. APVV-15-0116 and by the Slovak VEGA Grant 1/0368/16.

${ }^{3}$ Gefördert durch die Deutsche Forschungsgemeinschaft(DFG) - 327533333 und 270450205; partially supported by the grants 327533333 and SCHM 3186/1-1 (270450205) from the Deutsche Forschungsgemeinschaft (DFG, German Research Foundation), respectively.
} 
[1] M.B. Dillencourt, Polyhedra of small order and their Hamiltonian properties, J. Combin. Theory Ser. B 66 (1996) 87-122. doi:10.1006/jctb.1996.0008

[2] I. Fabrici, J. Harant and S. Jendrol, On longest cycles in essentially 4-connected planar graphs, Discuss. Math. Graph Theory 36 (2016) 565-575. doi:10.7151/dmgt.1875

[3] B. Grünbaum and J. Malkevitch, Pairs of edge-disjoint Hamilton circuits, Aequationes Math. 14 (1976) 191-196. doi:10.1007/BF01836218

[4] B. Jackson and N.C. Wormald, Longest cycles in 3-connected planar graphs, J. Combin. Theory Ser. B 54 (1992) 291-321. doi:10.1016/0095-8956(92)90058-6

[5] D.P. Sanders, On paths in planar graphs, J. Graph Theory 24 (1997) 341-345. doi:10.1002/(SICI)1097-0118(199704)24:4〈341::AID-JGT6〉3.0.CO;2-O

[6] A. Schmid and J.M. Schmidt, Computing Tutte paths (2017), arXiv-preprint. https://arxiv.org/abs/1707.05994

[7] C.-Q. Zhang, Longest cycles and their chords, J. Graph Theory 11 (1987) 521-529. doi:10.1002/jgt.3190110409

Received 16 October 2017

Revised 13 March 2018

Accepted 13 March 2018 